The 2012 ASCO (American Society of Clinical Oncology) annual meeting has been held once again at the McCormick Conference Center in Chicago, Illinois, where ASCO has booked a 10-year run for the meeting. The meeting was attended by more than 30,000 oncology professionals from around the world. Of more than 4500 abstracts published at the meeting, 310 were related to melanoma. Here we report the results of the most interesting clinical trials presented at the meeting. Apart from updated overall survival (OS) results of a phase 3 study evaluating the efficacy of vemurafenib and some new data on ipilimumab (expanded access program [EAP] and treatment of patients with brain metastases) we report on practice changing trials: a phase 3 (BREAK) trial evaluating efficacy of dabrafenib and a phase 3 study (METRIC) assessing trametinib in the treatment of metastatic melanoma patients. Another encouraging treatment strategy is combination of dabrafenib and trametinib evaluated in a phase I/II study. Results of new immune checkpoint targeting by monoclonal antibody anti-PD1 (BMS-936558) in an early phase trial in monotherapy or in combination with a multipeptide vaccine in metastatic melanoma patients are presented. Also, results of dendritic cell-based vaccine (randomized phase II trial) immunization in patients with high risk resected melanoma are shown. Furthermore, results of other melanoma immunotherapy strategies evaluated in early phase studies are reported.

Key words: melanoma, kinase inhibitors, BRAF inhibitor, MEK inhibitor, immunotherapy, cancer vaccines.

\section{Recent advances in melanoma treatment - American Society of Clinical Oncology (ASCO) 2012 perspective}

\author{
Jacek Mackiewicz' ${ }^{1}$ Andrzej Mackiewicz ${ }^{1,2}$
}

1Department of Cancer Immunology, Chair of Medical Biotechnology, Poznan University of Medical Sciences, Greater Poland Cancer Center, Poznan, Poland 2BioContract Sp. z o.o. Poznan, Poland

\section{Kinase inhibitors}

Vemurafenib is a selective BRAF inhibitor. Recently it has been evaluated in metastatic melanoma patients with BRAF V600E mutation after progression of earlier systemic treatment (phase 2 trial BRIM2). Vemurafenib was also tested in a phase 3 trial (BRIM3) in previously untreated patients. The results of these two trials led to its approval by the U.S. Food and Drug Administration (FDA) in August 2011 and the European Medicines Agency (EMA) in February 2012 for the treatment of metastatic melanoma with BRAF mutation. Updated overall survival (OS) results of the BRIM3 study have been presented at the ASCO meeting this year. The overall response rate in patients treated with vemurafenib was 57\% [5.6\% - complete response (CR), 51.3 - partial response $(\mathrm{PR})]$ compared with $8.6 \%(1.2 \%-C R, 7.4 \%-P R)$ observed in patients receiving dacarbazine (DTIC). Median progression-free survival (PFS) was also longer in patients treated with the study drug (6.9 vs. 1.6 months; HR 0.38; $95 \% \mathrm{Cl}: 0.32-0.46 ; p<0.001$ ) as well as median OS (13.6 vs. 9.7 months; HR $0.70 ; 95 \% \mathrm{Cl}: 0.57-0.87 ; p<0.001)$. In patients treated with vemurafenib, adverse cutaneous skin carcinoma, keratoacanthoma and skin papilloma were noted respectively in $19 \%, 11 \%$ and $28 \%$ of patients [1].

James Larkin presented results of an open-label, multicenter safety study of vemurafenib in patients with metastatic melanoma. Of 1964 screened patients, 914 were enrolled in the study and 834 evaluable for toxicity analysis. $70 \%$ of patients received prior systemic treatment due to metastatic melanoma (14\% - ipilimumab, $2 \%$ - MEK and BRAF inhibitors). Adverse events (AEs) were observed in $66 \%$ of patients ( $88 \%$ were related to vemurafenib). The most frequently observed any grade AEs were arthralgia (31\%), rash (29\%), fatigue (22\%), photosensitivity (21\%) and nausea (15\%). Grade 3 and 4 AEs occurred respectively in $33 \%$ and $1.9 \%$. The most common were rash (3.6\%), arthralgia (3.1\%) and cutaneous cell carcinoma/keratoacanthoma (4.3\%). In $6 \%$ of patients treatment was discontinued due to AEs (mainly arthritis and abdominal pain). At the time of study analysis 302 patients were evaluable for tumor assessment at week 8 of treatment; $61 \%$ developed objective responses, and 29\% stable disease (SD) [2].

Another active BRAF kinase inhibitor in the treatment of metastatic melanoma patients is dabrafenib (GSK2118436). Results of a randomized, open-label, multicenter phase 3 study (BREAK-3) comparing the efficacy of dabrafenib and DTIC in patients with BRAF V600E mutated metastatic melanoma were presented at the meeting. The study enrolled 250 previously untreated patients. In one study arm patients received oral dabrafenib at a dose of $150 \mathrm{mg}$ twice a day. In the second arm DTIC at a dose of $1000 \mathrm{mg} / \mathrm{m}^{2}$ was administered in three-week intervals. 31\% of patients presented greater than 
1 ECOG performance status and $66 \%$ of enrolled patients had stage M1c melanoma. The objective response rate was superior in patients treated with dabrafenib - 53\% vs. $19 \%$. Patients treated with the study drug also demonstrated longer median PFS - 5.1 vs. 2.7 months (HR 0.30; 95\% Cl: 0.18-0.53; $p<0.0001)$. The OS data were immature at this point for the study analysis. The most frequent adverse events observed in patients treated with dabrafenib were hyperkeratosis (37\%), headache (32\%), pyrexia (28\%), arthralgia (27\%) and skin papillomas (24\%). Serious adverse events (SAEs) included pyrexia (6\%), squamous cell carcinoma (6\%) and new primary melanoma (2\%) [3].

A number of clinical trials showed low efficacy of anti-cancer agents in melanoma patients with brain metastases. However, small molecules have demonstrated some efficacy in patients with solid tumors with concomitant brain metastases. Dabrafenib in a phase 2 study (BREAK-MB) in patients with BRAF V600E/K mutation with intracranial lesions demonstrated high clinical efficacy. The study enrolled stage IV melanoma patients with $\geq 1$ intracranial metastases. 127 patients were recruited to one of the two study arms, but only 41 patients reached 8-week disease assessment at the time of interim analysis. Patients in cohort A did not receive any prior brain metastasis treatment before entering the trial. Patients in group B before enrolment developed intracranial progression following prior brain therapy. Unconfirmed overall intracranial response rate (OIRR) was 53\% in patients with BRAF V600E mutant in both study cohorts. The unconfirmed OIRR in patients with BRAF V600K mutation was $20 \%$ and $50 \%$, respectively, in arms A and B. These preliminary results confirm efficacy of dabrafenib in melanoma patients with intra- and extracranial metastases with acceptable toxicity [4].

Trametinib is a reversible, highly selective allosteric inhibitor of MEK $1 / 2$ activation and kinase activity. Results of a phase 3 (METRIC) study comparing trametinib with chemotherapy in patients with BRAF V600/K mutant advanced or metastatic melanoma were presented at the ASCO conference. 273 patients were randomized in a $2: 1$ ratio to receive trametinib or paclitaxel/DTIC. Patients with disease progression after chemotherapy were allowed to cross over to the trametinib arm. The overall response rate observed in the study drug arm was $24 \%$ compared with $7 \%$ in patients treated with chemotherapy. In the group receiving trametinib the median PFS was greater than in the control arm - 4.8 vs. 1.4 months (HR 0.44; 95\% Cl: 0.31-0.64; $p<0.0001$ ). Analysis of OS demonstrated that $81 \%$ of patients in the trametinib group were alive after 6 months of followup, compared with 67\% in the chemotherapy group (HR 0.53; 95\% Cl: 0.30-0.94, $p<0.01)$. The most common adverse events observed in patients treated with trametinib were skin rash, diarrhea, edema, hypertension and fatigue. Typical adverse events connected with MEK inhibitor treatment included chorioretinopathy $(<1 \%)$ and decreased ejection fraction $(7 \%)$ [5].

Jeffrey Weber has presented encouraging results of a phase I/II study presenting safety and efficacy of dabrafenib combined with trametinib in BRAF V600 mutant metastatic melanoma patients. The trial enrolled 77 patients. The over- all response rate observed in the study was $56 \%$ with 4 CR, 39 PR, 29 SD and 3 PD. Overall PFS was 7.4 months. The most frequently noted SAEs were pyrexia (6.5\%), fatigue (6.5\%) and dehydration (6.5\%). The combination of dabrafenib and trametinib was associated with a lower incidence of MEK inhibitor related rash and BRAF inhibitor induced hyperproliferative skin lesions when compared to the single agents. Skin toxicity over grade 2 occurred only in $2 \%$ of patients. Cutaneous squamous cell carcinoma and keratoacanthoma were observed in $2 \%$ of patients [6].

Encouraging results of a Chinese phase II trial comparing first line treatment of rh-endostatin (angiogenesis inhibitor) plus DTIC with DTIC alone in patients with advanced melanoma have been presented. The study enrolled 110 patients. Patients treated with rh-endostatin plus DTIC showed longer PFS - 5.0 vs. 1.5 months (95\% Cl: $2.45-$ $7.55 ; p=0.004)$ and longer OS -16 vs. 7 months ( $95 \% \mathrm{Cl}$ : $10.46-21.54 ; p=0.003)$. However, no statistical difference in overall response rate between study groups was noted. Treatment with rh-endostatin combined with DTIC was well tolerated, with grade 3-4 toxicity (mainly elevated transaminase and thrombocytopenia) observed only in $1.7 \%$ of patients [7].

A new agent, cabozantinib, has been tested in a randomized phase II trial in patients with metastatic cutaneous/mucosal (70\%) and ocular (30\%) melanoma. Cabozantinib is an MET and VEGFR2 inhibitor. The study randomized 77 previously treated patients with a known BRAF mutation in $32 \%$. An objective response was observed in $60 \%$ of patients. Progression-free survival was longer in patients treated with cabozantinib -5.7 vs. 3.0 months ( $\mathrm{HR}=0.3 ; p=0.055)$. Toxicity of the study drug was comparable to that of other VEGFR (vascular endothelial growth factor receptor) TKIs (tyrosine kinase inhibitors) [8].

Interim analysis of a single arm phase II trial assessing another VEGFR TKI, pazopanib, and paclitaxel in first-line treatment for unresectable stage III and IV melanoma demonstrated a $48 \%$ overall response rate. Disease control (PR + + SD) was seen in $80 \%$ of patients. Grade 3 and 4 hypertension was observed in $28 \%$, transaminitis in $21 \%$, neutropenia in $14 \%$ of patients [9].

Studies of mTOR (mammalian target of rapamycin kinase) inhibitors in two small phase II studies demonstrated efficacy. In one trial in advanced melanoma patients temsirolimus was combined with bevacizumab and showed PR in $19 \%$ and SD in $56 \%$ of patients; the most notable responses were seen in patients with BRAF wild type tumors [10]. Another trial enrolled metastatic melanoma patients to evaluate the combination of everolimus with paclitaxel and carboplatin. Partial response was observed in 17\% and SD in $60 \%$ of patients [11].

\section{Immunotherapy}

Ipilimumab is a fully human monoclonal antibody (mAb) targeting cytotoxic T-lymphocyte antigen-4 (CTLA-4). Currently ipilimumab and vemurafenib are the only two new drugs approved for the treatment of metastatic melanoma. Ipilimumab's first approval was in the U.S. (2010) and subsequently in Europe (2011) in the second line treatment after 
failure of chemotherapy (MDX010-20 study). Ipilimumab is also accepted in the U.S. (2010) in previously untreated patients with metastatic melanoma.

During the ASCO meeting Omid Hamid presented the results of the ipilimumab U.S. expanded access program (EAP) in patients with unresectable stage III or IV melanoma. The study also enrolled patients with brain metastases (27\%), ocular melanoma (5\%) and mucosal melanoma (4\%). 906 patients were treated with ipilimumab in a dose of $10 \mathrm{mg} / \mathrm{kg}$ every 3 weeks (4 doses - induction phase) followed by $10 \mathrm{mg} /$ kg every 12 weeks (maintenance phase) until progression or unacceptable/unmanageable toxicity of the treatment. Durable OS over 3 years was observed in 17\% of patients. Drug-related SAEs were noted in $27 \%$. The most common were diarrhea (10\%), colitis (8\%), endocrinopathies (4\%), and dermatitis (0.8\%). Incidence of SAEs such as hepatitis (0.24\%), intestinal perforations $(0.36 \%)$ and drug-related death $(0.24 \%)$ was consistent with that seen in other trials evaluating $10 \mathrm{mg} / \mathrm{kg}$ ipilimumab monotherapy. $11 \%$ of patients discontinued treatment due to drug toxicity [12].

Currently a phase I/II trial evaluating ipilimumab in combination with vemurafenib is ongoing with the first enrolled metastatic melanoma (BRAF V600 mutant) patient in November 2011 [13].

Paul Chapman's group demonstrated interesting results of hypersensitivity skin reactions in melanoma patients treated with vemurafenib after previous ipilimumab therapy. The investigators observed drug-related rash associated with vemurafenib in 13 out of 16 treated patients (81\%). Four patients developed grade 3 maculopapular rash which occurred within 8 days of starting the vemurafenib treatment. Biopsies demonstrated spongiotic and perivascular dermatitis with eosinophils consistent with a drug hypersensitivity reaction. They did not progress to life-threatening reactions such as anaphylaxis or Stevens-Johnson syndrome and did not necessitate discontinuation of vemurafenib treatment. The incidence of grade 3 rash was higher than that observed in patients treated with vemurafenib in the phase III BRIM3 trial (25\% vs. $8 \% ; p=0.02)$ [14].

Ipilimumab has also shown some activity in patients with metastatic melanoma and brain metastases, particularly when metastases were small and asymptomatic. Results of a phase 2 study of these patients were published recently in Lancet Oncology. The intracranial response rate was 24\% (patients neurologically asymptomatic without corticosteroid treatment) and 10\% (patients with neurological symptoms on a stable dose of corticosteroids) [15]. Activity of ipilimumab and fotemustine in metastatic melanoma patients has been tested in the NIBIT-M1 trial. 86 patients with asymptomatic brain metastases were enrolled in the study (7 patients had prior whole brain radiotherapy or radiosurgery). Patients received 4 doses of ipilimumab $10 \mathrm{mg} / \mathrm{kg}$ every 3 weeks plus fotemustine $100 \mathrm{mg} / \mathrm{m}^{2}$ weekly for 3 weeks, followed by ipilimumab every 12 weeks (from week 24) combined with fotemustine every 3 weeks (from week 9). The immune related (ir) disease control rate (irDCR $=C R+P R+$ + SD using the ir response criteria) was 50\% and immune related overall response rate (irORR $=C R+P R$ ) was 40\%. Median irPFS was 4.6 months and 1-year OS was $52 \%$. Median OS was not reached at the time of study analysis. Grade
3 or 4 toxicity was observed in $60 \%$ of patients (hematological toxicity - 50\%, elevated ALT/AST - 5\%, gastrointestinal adverse events - 5\%) [16].

Another trial evaluated the development of brain metastases in metastatic melanoma patients treated with ipilimumab and temozolomide. Of 64 enrolled patients ( 2 with a history of prior brain metastases), 11 (17\%) developed brain metastases at a median follow-up of 41 weeks. The median time to brain metastases expansion was 12 weeks [17].

A new interesting anti-cancer agent is anti-PD-1 (BMS936558, MDX-1106), a fully human mAb that blocks the programmed death-1 (PD-1) co-inhibitory receptor expressed by activated T-cells. BMS-936558 was evaluated in 95 previously treated metastatic melanoma patients. The study drug was administered intravenously every 2 weeks until PD or CR, for a maximum of 12 cycles. The doses varied depending on the study cohort (0.1-10 mg/kg). SAEs occurred in 19\% of patients. The most common included gastrointestinal (4\%), endocrine (2\%) and hepatobiliary disorders (1\%). The observed response rate was $20-41 \%$ depending on the study cohort. Of the 20 patients that responded to the treatment, 12 developed a response lasting over 1 year. Currently further evaluation of BMS-936558 is ongoing [18]. BMS936558 was also evaluated in combination with a multipeptide vaccine in a phase I study in 30 previously treated patients with metastatic melanoma. Patients were vaccinated with MART-1/gp100/NY-ESO-1 peptides with adjuvant Montanide ISA 51 together with BMS-936558 (1, 3 or 10 mg/kg) every 2 weeks for 24 weeks, followed by BMS-936558 alone every 3 months. Responses to the treatment were observed in all study cohorts $(1 / 3 / 10 \mathrm{mg} / \mathrm{kg}-2 \mathrm{PR} / 5 \mathrm{PR} / 2$ $P R$ and $1 S D)$. Immunological tests demonstrated decreased PD-1 receptor on CD4+ and CD8+ lymphocytes and increased CTLA4 receptors, and a dose-related decrease in CD8 T-cells while CD4 T-cells increased [19].

Investigators from Russia presented results of a trial evaluating efficacy of a dendritic cell (DC)-based vaccine in patients with high risk resected melanoma (stage III and IV). 108 patients were enrolled in the trial. In one study arm 56 patients (stage III - 46, IV - 10) were treated with autologous monocyte-derived DC vaccine primed with autologous tumor lysate, administered intradermally every 2-6 weeks until disease progression. In the control group 53 patients (stage III - 47, IV - 5) underwent observation. At a median follow-up of 22 months disease-free survival (DFS) was significantly longer in patients treated with the vaccine (HR 0.45; 95\% Cl: 0.29-0.69; $p<0.05)$ although there was no difference in OS between the study arms (HR 0.71; 95\% Cl: $0.40-$ $1.25 ; p=0.23) .60 \%$ of vaccinated patients remained alive at the time of study analysis. The investigators observed a significant correlation between reduction of risk and vaccineinduced strong delayed type hypersensitivity reaction. The vaccine was safe and well tolerated [20].

At the ASCO meeting investigators reported on a phase 3 trial (OPTiM) evaluating effectiveness of intratumoral injections of talimogene laherparepvec (T-VEC) compared with subcutaneous administration of GM-CSF (granulocyte-macrophage colony-stimulating factor) in patients with advanced and metastatic melanoma. T-VEC (formally OncoVEXGM-SCF) is an oncolytic HSV1 that selectively replicates in tumors induc- 
ing a systemic anti-tumor immune response enhanced by local GM-SCF expression. Results of this trial are expected later in 2012 [21].

An interesting strategy consisting of CD40 ligand/interferon- $\gamma$ matured autologous DC immunized with gp100 antigen HLA (human leukocyte antigen) I restricted peptides in patients with metastatic melanoma has been shown. Out of 7 treated patients 2 PR were noted and 1 CR with a duration of over 3 years. 6 patients developed CD8+ T cell immunity to gp100. The investigators demonstrated a significant correlation of IL-12 production by DCs with TTP (time to progression) [22]. Other early phase immunotherapy trials included: intra-lesional administration of TG1042 (adenovirus expressing interferon-gamma) combined with adoptive TIL (tumor infiltrating lymphocytes) transfer [23]; TCR-IL-2 (T-cell receptor-interleukin-2) fusion protein in combination with cisplatin [24]; HyperAcute-Melanoma vaccine combined with pegylated interferon [25]; and autologous TIL infusion combined with lymphodepleting chemotherapy and low-dose IL-2 [26].

\section{References}

1. Chapman PB, Hauschild A, Robert C, et al. Updated overall survival (OS) results for BRIM-3, a phase III randomized, open-label, mul ticenter trial comparing BRAF inhibitor vemurafenib (vem) with dacarbazine (DTIC) in previously untreated patients with BRAFV600E-mutated melanoma. J Clin Oncol 2012; 30 (suppl): abstr. 8502.

2. Larkin JMG, Queirolo P, Aranc AM, et al. An open-label, multicenter safety study of vemurafenib (PLX4032, RO5185426) in patients with metastatic melanoma. J Clin Oncol 2012; 30 (suppl): abstr. 8517.

3. Hauschild A, Grob JJ, Demidov LV, et al. Phase III, randomized, openlabel, multicenter trial (BREAK-3) comparing the BRAF kinase inhibitor dabrafenib (GSK2118436) with dacarbazine (DTIC) in patients with BRAFV600E-mutated melanoma. J Clin Oncol 2012; 30 (suppl): abstr. LBA8500.

4. Kirkwood JM, Long GV, Trefzer U, et al. BREAK-MB: A phase II study assessing overall intracranial response rate (OIRR) to dabrafenib (GSK2118436) in patients (pts) with BRAF V600E/k mutation-positive melanoma with brain metastases (mets). J Clin Oncol 2012 30 (suppl): abstr. 8501

5. Robert C, Flaherty KT, Hersey P, et al. METRIC phase III study: Efficacy of trametinib (T), a potent and selective MEK inhibitor (MEKi), in progression-free survival (PFS) and overall survival (OS), compared with chemotherapy $(\mathrm{C})$ in patients (pts) with BRAFv600E/K mutant advanced or metastatic melanoma (MM). J Clin Oncol 2012; 30 (suppl): abstr. LBA8509.

6. Weber JS, Flaherty KT, Infante JR. Updated safety and efficacy results from a phase $1 /$ II study of the oral BRAF inhibitor dabrafenib (GSK2118436) combined with the oral MEK 1/2 inhibitor trametinib (GSK1120212) in patients with BRAFi-naive metastatic melanoma. J Clin Oncol 2012; 30 (suppl): abstr. 8510.

7. Guo J, Cui CL, Gu K, et al. Randomized, double-blind, and multicenter phase II trial of rh-endostatin plus dacarbazine versus dacarbazine alone as first-line therapy for the patients with advanced melanoma. J Clin Oncol 2012; 30 (suppl): abstr. 8554

8. Gordon MS, Kluger HM, Shapiro G, et al. Activity of cabozantinib (XL184) in metastatic melanoma: Results from a phase II randomized discontinuation trial (RDT). J Clin Oncol 2012; 30 (suppl): abstr. 8531

9. Fruehauf JP, Alger B, Parmakhtiar B, et al. A phase II single arm study of pazopanib and paclitaxel as first-line treatment for unresectable stage III and stage IV melanoma: Interim analysis. J Clin Oncol 2012; 30 (suppl): abstr 8524

10. Slingluff CL, Petroni GR, Molhoek KR, et al. Clinical activity and safety of combination therapy with temsirolimus and bevacizumab for advanced melanoma: Phase II trial with correlative studies. J Clin Oncol 2012; 30 (suppl): abstr. 8530.
11. Hauke RJ, Infante JR, Shih KC, et al. Everolimus in combination with paclitaxel and carboplatin in patients with advanced melanoma: A phase II trial of the Sarah Cannon Research Institute (SCRI). J Clin Oncol 2012; 30 (suppl): abstr. 8556.

12. Hamid O, Hwu WJ, Richards JM, et al. Ipilimumab (Ipi) expanded access program (EAP) for patients (pts) with stage III/IV melanoma: 10 mg/kg cohort interim results. J Clin Oncol 2012; 30 (suppl): abstr. 8508.

13. Ribas A, Hodi FS, Kurland JF, et al. CA184-161: A phase I/II trial of vemu rafenib and ipilimumab in patients with BRAF V600 mutation-positive metastatic melanoma. J Clin Oncol 2012; 30 (suppl): abstr. TPS8603.

14. Harding JJ, Lacouture ME, Pulitzer M, et al. Hypersensitivity skin reactions in melanoma patients treated with vemurafenib after ipilimumab therapy. J Clin Oncol 2012; 30 (suppl): abstr. 8515.

15. Margolin K, Ernstoff MS, Hamid O, et al. Ipilimumab in patients with melanoma and brain metastases: an open-label, phase 2 trial. Lancet Oncol 2012; 13: 459-65.

16. Maio M, Testori A, Ascierto PA, et al. The NIBIT-M1 trial: Activity of ipilimumab plus fotemustine in patients with melanoma and brain metastases. J Clin Oncol 2012; 30 (suppl): abstr. 8529.

17. Wang J, Patel SP, Hwu WJ, et al. Development of brain metastases in patients with metastatic melanoma treated with ipilimumab plus temozolomide. J Clin Oncol 2012; 30 (suppl): abstr. e19014.

18. Hodi FS, Sznol M, McDermott DF, et al. Clinical activity and safety of anti-PD-1 (BMS-936558, MDX-1106) in patients with advanced melanoma (MEL). J Clin Oncol 2012; 30 (suppl): abstr. 8507.

19. Kudchadkar RR, Gallenstein D, Martinez AJ, et al. Phase I trial of extended-dose anti-PD-1 antibody BMS-936558 with a multipeptide vaccine for previously treated stage IV melanoma. J Clin Oncol 2012; 30 (suppl): abstr. 8582.

20. Petenko NN, Mikhaylova IN, Chkadua GZ, et al. Adjuvant dendritic cell (DC)-based vaccine therapy of melanoma patients. J Clin Oncol 2012; 30 (suppl): abstr. 2524.

21. Kaufman H, Wagner VJ, Goldsweig H, Yao B, Coffin R. OPTiM: A randomized phase III trial to evaluate the efficacy and safety of talimogene laherparepvec (T-VEC) compared with subcutaneously (sc) administered GM-CSF for the treatment (tx) of unresectable stage IIIb, IIIc, and IV melanoma. J Clin Oncol 2012; 30 (suppl): abstr. TPS8604.

22. Linette GP, Becker-Hapak M, Huang A, et al. CD40 ligand/interferon$\gamma$ matured DC immunization with gp100 antigen HLA class I A *0201 restricted peptides in patients with newly diagnosed metastatic melanoma. J Clin Oncol 2012; 30 (suppl): abstr. 2525.

23. Khammari A, Limacher JM, Nguyen JM, et al. Intra-lesional administrations of TG1042 (adenovirus expressing interferon- $\gamma$ ) combined with adoptive TIL transfer in patients with metastatic melanoma. J Clin Oncol 2012; 30 (suppl): abstr. e19019.

24. Milhem MM, Weber JS, Amin A, et al. Clinical experience of a targeted TCR-IL2 fusion protein in combination with cisplatin (CDDP) in patients (pts) with metastatic melanoma. J Clin Oncol 2012; 30 (suppl): abstr. e13088.

25. Riker Al, Rossi GR, Alsfeld LC, et al. Final results of a phase II immunotherapy trial for stage III and IV melanoma patients. J Clin Oncol 2012; 30 (suppl): abstr. e19008.

26. Ellebaek E, Iversen TZ, Junker N, et al. Adoptive cell therapy for melanoma patients using tumor-infiltrating lymphocytes, lymphodepleting chemotherapy, and low-dose interleukin-2. J Clin Oncol 2012; 30 (suppl): abstr. 2574.

\section{Address for correspondence}

Jacek Mackiewicz MD, PhD

Department of Cancer Immunology

Greater Poland Center

Garbary 15

61-866 Poznań, Poland

e-mail: jmackiewicz@biocontract.com

Submitted: $\quad 15.06 .2012$

Accepted: $\quad 20.06 .2012$ 